\title{
Quasicyclic Dyadic Codes in Walsh-Hadamard Domain
}

\author{
B. Sundar Rajan ${ }^{1}$ \\ Dept. of Elect. Comm. Engg. \\ Indian Institute of Science \\ Bangalore 560012, India \\ e-mail: bsrajan@ece. \\ iisc.ernet.in
}

\begin{abstract}
A dyadic code is a code which is closed under all dyadic shifts. A quasicyclic dyadic (QCD) code is one which is both quasicyclic and dyadic. We obtain a simple characterization of all QCD codes over any field of odd characterisic using Walsh-Hadamard Transform defined over that field.
\end{abstract}

\section{Preliminaries}

An $s$-quasicyclic code is a code with the property that cyclic shift of any codeword by $s$ positions gives another codeword. Cyclic codes are 1-quasicyclic codes. Dyadic codes are defined for length $n=2^{r}$ as follows: For any integer $i \in\left\{0,1, \ldots, 2^{r}-1\right\}$, let $\lceil i\rceil=\left\lceil i_{r-1}, i_{r-2}, \ldots, i_{1}, i_{0}\right\rceil$ denote its radix- 2 representation, where

$$
i=i_{r-1} 2^{r}+i_{r-2} 2^{r-1}+\ldots+i_{1} 2+i_{0},
$$

and $i_{j}=0,1$ for $j=0,1, \ldots, r-1$. Addition of two numbers $\lceil i\rceil$ and $\lceil j\rceil$, denoted by $\lceil i\rceil \oplus\lceil j\rceil$ is defined by $\lceil i\rceil \oplus\lceil j\rceil=\lceil k\rceil$, where $k_{l}=\left(i_{l}+j_{l}\right)$ modulo 2 , for $l=0,1 \ldots,(r-1)$. By an $m$-dyadic shift, $m=0,1, \ldots, n-1$, of a vector $\left(a_{0}, a_{1}, \ldots, a_{n-1}\right)$ is meant the vector $\left(a_{0 \oplus m}, a_{1 \oplus m}, \ldots, a_{(n-1) \oplus m}\right)$ [1].

Definition 1: A linear code of length $n=2^{r}$ over a field is said to be a dyadic code if $m$-dyadic shift of every codeword is also a codeword for all $m=0,1 \ldots, n-1$.

Throughout the correspondence, we will consider linear codes over a finite field with $q$ elements, denoted by $F_{q}$, where $q$ is a power of an odd prime $p$.

Definition 2: A dyadic code of length $n=2^{r}$ is said to be a Quasi-Cyclic Dyadic (QCD) code if is an $t$-quasicyclic. If $t$ is the smallest such interger, say $t=2^{s}$ for some $s=0,1, \ldots, r-1$, we call the code a " $2^{s}$-QCD code". Note that a $2^{0}$-QCD code is a cyclic code which is also dyadic.

We shall characterize all $2^{s}$-QCD codes in the transform domain using the Walsh-Hadamard transform (WHT) defined over $F_{q}$. Our characterization does not need extension of the field. Notice that if a cyclic code over $F_{q}$ of length $n$ is to be described in the transform domain using DFT then it is necessary that the DFT is defined in the extension field $F_{q^{m}}$, where $m$ is the least integer such that $n$ divides $q^{m}-1$ [2]. The main result of this correspondence is that if such a cyclic code is dyadic also, then using WHT it can be described without field extension.

Definition 3: Let $\left(a_{0}, a_{1}, \ldots, a_{n-1}\right)$ be a $n$-length vector over $F_{q}$, a field with odd characteristic $p$ and $n=2^{r}$. The WHT of this vector is defined to be the $n$-length vector $\left(A_{0}, A_{1}, \ldots, A_{n-1}\right)$ over $F_{q}$, given by

$$
A_{j}=\sum_{i=0}^{n-1}(-1)^{<j, i>} a_{i} ; \quad j=0,1, \ldots, n-1
$$

${ }^{1}$ This work was partly supported by CSIR, India, through Research Grant (22(0298)/99/EMR-II) to B. S. Rajan

\author{
Moon Ho Lee \\ Institute of Information and \\ Communication \\ Chonbuk National University \\ Chonju, Korea \\ e-mail: moonho@chonbuk.ac.kr
}

where $\langle j, i\rangle$ denotes the modulo-2 inner product

$$
j_{r-1} i_{r-1}+j_{r-2} i_{r-2}+\ldots+j_{0} i_{0} \quad(\operatorname{modulo} 2) .
$$

\section{QCD Codes IN WHT Domain}

In this section we present the main result of this correspondence which gives the constraints among the spectral components to be satisfied for a dyadic code to be a $2^{s}-\mathrm{QCD}$ code. Obviously,

Theorem 1: All length $n=2^{r}$ dyadic codes are $2^{r-1}$-QCD codes.

Definition 4: A subset $\mathcal{S}$ of spectral components $\left\{A_{0}, A_{1}, \cdots, A_{n-1}\right\}$ is said to be a constrained set of spectral components for a dyadic code $\mathcal{C}$, if any one spectral component in $\mathcal{S}$ takes only the zero value then all other spectral components in $\mathcal{S}$ also take only the zero value for $\mathcal{C}$. A constrained set with only one spectral component will be referred as a free spectral component or equivalently a spectral component which does not belong to any constrained set with at least two elements is a free spectral component.

Definition 5: For a given $s, 0 \leq s \leq r-2$, for every $\mu$, $(s+1 \leq \mu \leq r-1)$ and a fixed $\left(\bar{j}_{s-1}, \bar{j}_{s-2}, \ldots, j_{0}\right)$, the set of $2^{\mu-s}$ spectral components consisting of those $A_{j}$ 's where

$j=\left\lceil 0,0, \ldots, 0,1=j_{\mu}, j_{\mu-1}, j_{\mu-2}, \ldots, j_{s+1}, j_{s}, j_{s-1}, \ldots, j_{0}\right\rceil$

is denoted by $J\left(\mu, s, j_{s-1}, j_{s-2}, \ldots, j_{0}\right)$.

The main result of this correspondence is

Theorem 2: A dyadic code $\mathcal{C}$ of length $n=2^{r}$ is $2^{s}$-QCD, $0 \leq s<r-2$, iff the set of WHT vectors of all the codewords satisfy the following conditions:

(i) For all $0 \leq j \leq 2^{s+1}-1$, the spectral component $A_{j}$ is free.

(ii) Among all $j \geq 2^{s+1}$, every $J\left(\mu, s, j_{s-1}, j_{s-2}, \ldots, j_{0}\right)$ form a constrained set of spectral components for $\mathcal{C}$.

Example 1: For length 8 codes over any field of odd characteristic, there are $2^{4}=16$ cyclic dyadic codes corresponding to the following 4 constrained sets of spectral components. $\left\{A_{0}\right\} \quad\left\{A_{1}\right\} \quad\left\{A_{2}, A_{3}\right\} \quad\left\{A_{4}, A_{5}, A_{6}, A_{7}\right\}$.

There are $2^{6}=64$ number of 2-QCD codes corrsponding to the following 6 constrained sets of spectral components: $\left\{A_{0}\right\} \quad\left\{A_{1}\right\} \quad\left\{A_{2}\right\} \quad\left\{A_{3}\right\} \quad\left\{A_{4}, A_{6}\right\} \quad\left\{A_{5}, A_{7}\right\}$.

\section{REFERENCES}

[1] Ahmed N. and Rao K.R., Orhtogonal Transforms for Digital Signal Processing, Springer-Verlag, New York, 1975.

[2] Blahut R.E., Theory and Practice of Error Control Codes, Addition-Wesley, 1982, 